\title{
INDÚSTRIA 4.0: TECNOLOGIAS E NÍVEL DE MATURIDADE DE SUAS
}

\section{APLICAÇÕES}

Tatiana Domingues de Almeida (tatiana.almd@gmail.com) -Universidade Federal de Santa

Catarina

Caroline Gobbo Sá Cavalcante (carolinegobbosa@gmail.com) -Universidade Federal de Santa Catarina

Diego de Castro Fettermann (d.fettermann@ufsc.br) -Universidade Federal de Santa Catarina

\section{RESUMO}

A utilização de tecnologias integradoras na manufatura direcionou a indústria para um novo paradigma de produção, em que existem menores interferências humanas e o sistema é inteligente e interconectado, a Indústria 4.0. Este sistema possui foco na produção inteligente de produtos, métodos e processos. Tecnologias como RFID, sensores, processamento na nuvem, impressões 3D fazem parte dos muitos componentes que podem ser encontrados em um sistema orientado pela Indústria 4.0. Este artigo tem por objetivo identificar as tecnologias e o nível de maturidade das aplicações da Indústria 4.0 nas empresas. A partir de um levantamento de 38 casos de sucesso resultantes de implementações tecnológicas associadas à Indústria 4.0 serão identificadas as tecnologias mais utilizadas e o atual nível de desenvolvimento destas tecnologias na prática das empresas. Os resultados indicam um nível inicial de desenvolvimento destas tecnologias nas empresas, indicando um potencial de aumento de produtividade com a aplicação destas tecnologias no setor produtivo.

Palavras chave: smart industry; Internet of Things; tecnologias; níveis de maturidade 


\section{INTRODUÇÃO}

A Indústria 4.0 reflete um novo paradigma no que diz respeito às grandes revoluções industriais. No século 18 ocorreu a primeira revolução industrial, em que começaram a ser introduzidas as máquinas nos processos industriais com o aprimoramento das máquinas a vapor e a criação do tear mecânico (LU, 2017). No início do século 20 estava em andamento uma nova revolução que se caracterizou pela divisão do trabalho, introdução da produção em massa com ajuda da energia elétrica, a exploração de novos materiais, como aço e produtos sintéticos e o descobrimento de novas formas de combustível (SHROUF, 2014; BRETTEL et al., 2014). Nos anos 70, a terceira revolução industrial passa a ser incorporada à indústria, caracterizada pela aplicação de controladores lógicos programáveis para automação da manufatura (BRETTEL et al., 2014) além da utilização de tecnologias da informação para o gerenciamento da produção (STOCK; SELIGER, 2016). Atualmente os processos produtivos se preparam para o que pode ser denominada uma quarta revolução industrial, viabilizada pela utilização da internet para permitir a comunicação entre pessoas e também entre máquinas em um sistema denominado Físico-Cibernético por meio de amplas redes de comunicação (BRETTEL et al., 2014). Este novo paradigma está embasado em fábricas digitalizadas, capazes de combinar tecnologias da internet e máquinas e produtos inteligentes (smarts) (LASI; KEMPER, 2014).

O desenvolvimento da Indústria 4.0 busca atingir um alto nível de eficiência operacional, produtividade e automação dos sistemas produtivos. Apesar do objetivo comum, a literatura não apresenta um consenso para a sua definição (SHAFIQ et al., 2015; LU, 2017). WEYER et al. (2015) propõe o conceito de Indústria 4.0 como dispositivos, máquinas, módulos de produção e produtos organizados em um sistema Físico-Cibernético com capacidade de trocar informações de forma autônoma e realizar autocontrole de suas operações (WEYER et al., 2015). Apesar disso, este conceito pode ainda ser ampliado para uma visão da cadeia de valor, como a um novo nível de organização e gerenciamento da cadeia de valor durante o ciclo de vida dos produtos ou ainda como um conjunto de tecnologias e conceitos aplicados na organização da cadeia de valor (HERMANN et al., 2016).

Assim como a diversidade dos conceitos de Indústria 4.0, os benefícios de sua aplicação também podem ser variados. A integração de objetos físicos, interações humanas, máquinas inteligentes, processos e linhas de produção resulta no desenvolvimento de uma nova cadeia de valor, inteligente, conectada e ágil (SCHUMACHER et al., 2016). Esta nova cadeia de valor viabiliza o desenvolvimento de novos modelos de negócios; com diferentes formas para a organização do trabalho (KAGERMANN et al., 2013). Estas possibilidades de arranjo das diferentes alternativas das tecnologias da Indústria 4.0 podem resultar em diversos benefícios, tais como: melhorias na redução do tempo de processamento dos produtos (QIN et al, 2016), melhorias de qualidade (LEE et al., 2015), eficiência dos processos (LASI; KAMPER, 2014), redução do custo de processos (QIN et al, 2016; STOCK; SELIGER, 2016), flexibilidade de processos (BRETTEL et al., 2014), melhor atendimentos dos requisitos dos clientes (STOCK; SELIGER, 2016), personalização dos produtos (BRETTEL et al., 2014; SHAFIQ et al., 2015) entre outros.

Este artigo tem por objetivo identificar as tecnologias e o nível de maturidade das aplicações da Indústria 4.0 nas empresas. São diversas as possibilidades dessas aplicações e a literatura é bastante genérica para identificar com precisão as contribuições da Indústria 4.0. Para tanto, foi realizado um levantamento de casos de sucesso dessas aplicações para verificar quais as tecnologias utilizadas assim como o nível de maturidade de implementação. Os resultados obtidos contribuem para identificar as características dos casos de aplicação da Indústria 4.0. 
Este resultado estabelece um panorama dessa aplicação prática e compreender o nível de implementação deste tipo de tecnologia. Permite mitigar a falta de procedimentos para a implementação da Indústria 4.0 nas empresas, tanto ressaltada na literatura (DRATH; HORCH, 2014; QIN et al., 2016).

\section{REFERENCIAL TEÓRICO}

Para atingir o objetivo foi realizada uma revisão sobre quais são tecnologias da Indústria 4.0 e sobre as classificações de modelos de maturidade propostos para Indústria 4.0.

\subsection{Tecnologias Indústria 4.0}

A Indústria 4.0 utiliza dispositivos e tecnologias que tornam possível o desenvolvimento de diversas oportunidades traduzidas em novos produtos ou serviços (TAMÁS et al., 2016). Essas aplicações são capazes de oferecer vantagens e proporcionam uma abordagem vasta tanto no nível técnico quanto no nível organizacional. Estas tecnologias podem contribuir de diversas formas a melhoria do desempenho do processo de manufatura (LEE et al., 2015). A aplicação das tecnologias da Indústria 4.0 permitem a transformação do gerenciamento das operações de manufatura, resultando em sua descentralização (SHAFIQ et al., 2015), uma maior integração vertical (ALMADA-LOBO, 2016) e horizontal (BRETTEL et al., 2014, HERMANN et al., 2016) da empresa, além do monitoramento remoto dos processos (ALMADA-LOBO, 2016).

Para implementar esta transformação estão disponíveis uma diversidade de tecnologias, muitas destas com objetivos semelhantes. A literatura não apresenta uma forma única para denominar as tecnologias da Indústria 4.0. Muitas das classificações de tecnologias utilizadas na literatura são inclusive incompatíveis entre si, classificando as mesmas tecnologias em categorias diferentes. Como forma de reduzir esta diversidade de classificação este trabalho utiliza as funcionalidades das tecnologias como forma de categorizá-las, resultando em sete diferentes classes. A primeira classe compreende tecnologias utilizadas para o processamento de informações, denominada de $(i)$ análise e processamento de dados, que incorpora tecnologias como de algoritmos avançados para otimização dos processos, machine learning, mineração de dados, big data e autenticação e detecção de fraudes. A segunda classe de tecnologias está direcionada para aumentar a percepção e uso das informações, denominada de (ii) realidade aumentada, compreendendo a inserção de objetos virtuais no ambiente físico, mostrada ao usuário em tempo real com o apoio de algum dispositivo tecnológico como wearables. A terceira classe compreende tecnologias para o processamento de dados remotamente, denominada de (iii) computação em nuvem. A quarta categoria, denominada de (iv) dispositivos móveis, compreende a utilização de terminais móveis para acesso às informações, tais como smartphones, tablets, terminais entre outros. A quinta categoria de tecnologias é denominada de (v) IoT, está destinada por realizar a comunicação e apresentação das informações, compreende a utilização de sensores inteligentes, middleware, tecnologias de detecção de localização, aplicativos IoT, interface de aplicativos (apps), RFID entre outros. O quinto tipo de tecnologias é denominada de ( $v i)$ manufatura aditiva, tais como impressoras 3D utilizando polímeros, metais, alimentos entre outros. Por fim, a sétima categorias de tecnologias, denominada de (vii) sistemas Físico-Cibernéticos, compreende a utilização de robotização, automação e a utilização avançada de interface homem-máquina, máquina-máquina. A partir desta definição foi possível identificar a frequência de citação destas tecnologias na literatura (Tabela 1). 
Tabela 1 - Citação de tecnologias voltadas à Indústria 4.0

\begin{tabular}{|c|c|c|c|c|c|c|c|c|c|}
\hline Tecnologias & $\begin{array}{c}\text { PwC } \\
(2016)\end{array}$ & $\begin{array}{l}\text { Lee e } \\
\text { Lee } \\
(2015)\end{array}$ & $\begin{array}{c}\text { Zuehlke } \\
\text { (2010) }\end{array}$ & $\begin{array}{l}\text { Tamás } \\
\text { et al. } \\
\text { (2016) }\end{array}$ & $\begin{array}{l}\text { Bagheri } \\
\text { et al. } \\
(2015)\end{array}$ & $\begin{array}{l}\text { Qin et } \\
\text { al. } \\
(2016)\end{array}$ & $\begin{array}{l}\text { Hozdić } \\
\text { (2015) }\end{array}$ & $\begin{array}{c}\text { CNI } \\
(2016)\end{array}$ & $\begin{array}{l}\text { Almada- } \\
\text { Lobo } \\
(2016)\end{array}$ \\
\hline $\begin{array}{c}\text { Análise e } \\
\text { processamento de } \\
\text { dados } \\
\end{array}$ & $\mathrm{X}$ & $\mathrm{X}$ & $\mathrm{X}$ & $X$ & $\mathrm{X}$ & & & $\mathrm{X}$ & $\mathrm{X}$ \\
\hline Realidade aumentada & $X$ & & & & & & & & \\
\hline Computação em nuvem & $X$ & $\mathrm{X}$ & & & & & & $\mathrm{X}$ & $\mathrm{X}$ \\
\hline Dispositivos móveis & $\mathrm{X}$ & & & & $\mathrm{X}$ & & & & $\mathrm{X}$ \\
\hline IoT & $X$ & $\mathrm{X}$ & $X$ & $X$ & $\mathrm{X}$ & $\mathrm{X}$ & $X$ & $\mathrm{X}$ & $\mathrm{X}$ \\
\hline Manufatura aditiva & $\mathrm{X}$ & & & & & & & $\mathrm{X}$ & $\mathrm{X}$ \\
\hline $\begin{array}{c}\text { Sistemas Físico- } \\
\text { Cibernéticos } \\
\end{array}$ & $X$ & & & $X$ & $\mathrm{X}$ & $X$ & $\mathrm{X}$ & $\mathrm{X}$ & $\mathrm{X}$ \\
\hline
\end{tabular}

\subsection{Modelos de Maturidade da Indústria 4.0}

O objetivo de um modelo de maturidade é descrever as etapas de melhoria de um processo desde um nível incipiente para um nível avançado, incorporando os componentes de cada nível e as recomendações para sua utilização (PAULK et al.,1993). Na literatura ainda são poucas as propostas de modelos de maturidade de implementação da Indústria 4.0 nas empresas (SCHUMACHER et al., 2016; PWC, 2016; ROCKWELL AUTOMATION, 2014; LANZA et al., 2015; PORTER; HEPPELMANN, 2015). Entre estas propostas, a desenvolvida por Schumacher et al. (2016) apresenta uma análise mais sistêmica da aplicação da Indústria 4.0 nas empresas, considerando 62 itens que mensuram por meio de uma escala Likert de 5 pontos o nível de implementação dos itens categorizados em nove diferentes dimensões. Cada uma das dimensões consideradas para a identificação dos níveis de maturidade de implementação da Indústria 4.0 nos modelos analisados são apresentadas na Tabela 2.

Tabela 2- Dimensões propostas em modelos de maturidade em Indústria 4.0.

\begin{tabular}{|c|c|c|c|c|c|}
\hline Áreas de avaliação & $\begin{array}{c}\text { Schumacher } \\
\text { et al. (2016) }\end{array}$ & $\begin{array}{c}\text { Lanza et al. } \\
(2016)\end{array}$ & $\begin{array}{c}\text { RwC (2016) } \\
\text { Automation } \\
(2014)\end{array}$ & $\begin{array}{c}\text { Porter e } \\
\text { Heppelmann } \\
(2015)\end{array}$ \\
\hline Estratégia & $\mathrm{X}$ & $\mathrm{X}$ & $\mathrm{X}$ & $\mathrm{X}$ & \\
\hline Liderança & $\mathrm{X}$ & & & & \\
\hline Consumidores & $\mathrm{X}$ & & $\mathrm{X}$ & & \\
\hline Produtos & $\mathrm{X}$ & & $\mathrm{X}$ & & $\mathrm{X}$ \\
\hline Operações & $\mathrm{X}$ & $\mathrm{X}$ & $\mathrm{X}$ & $\mathrm{X}$ & \\
\hline Cultura & $\mathrm{X}$ & & $\mathrm{X}$ & & \\
\hline Pessoas & $\mathrm{X}$ & & & $\mathrm{X}$ & \\
\hline Governança & $\mathrm{X}$ & & & & \\
\hline Segurança & & & $\mathrm{X}$ & $\mathrm{X}$ & \\
\hline Tecnologia & $\mathrm{X}$ & $\mathrm{X}$ & $\mathrm{X}$ & $\mathrm{X}$ & $\mathrm{X}$ \\
\hline
\end{tabular}

Verifica-se que a dimensão tecnologia é a mais frequente nos modelos de maturidade analisados, sendo orientada para a mensuração do nível de adoção das tecnologias referentes à Indústria 4.0 (SCHUMACHER et al., 2016; PWC, 2016; ROCKWELL AUTOMATION, 2014; LANZA et al., 2015; PORTER; HEPPELMANN, 2015). Apesar disso, os diversos 
modelos de maturidade incluem outras dimensões, muito em razão de considerar o aspecto sistêmico que a sistema da Indústria 4.0 apresenta nas organizações.

\section{MÉTODO}

\subsection{Fonte de dados}

A busca de casos de sucesso da implementação da Indústria 4.0 foi desenvolvida de forma a identificar de aplicações nas suas mais variadas áreas. Inicialmente foram identificados dados de apresentações realizadas em congressos da área como forma de garantir a distribuição dos casos nas mais diversas áreas de atuação. Foram avaliados sete congressos considerados relevantes para a área (LIAO et al., 2017). Seguindo recomendação foram analisados os casos, referente aos anos 2014, 2015 e 2016, apresentados nos seguintes congressos: IEEE International Conference on Emerging Technologies and Factory Automation (ETFA), CIRP Conference on Manufacturing Systems (CIRP - CMS), Internet of Things World Forum, IEEE International Conference on Industrial Informatics (INDIN) e IFAC Symposium on Information Control Problems in Manufacturing (INCOM). Ao final deste procedimento foi possível identificar somente dois casos com a identificação da empresa e informações suficientes para posterior análise. Também foram coletados seis casos a partir da informação obtida nas apresentações das empresas nestes congressos. Os dados destes casos foram aprofundados a partir das informações coletadas diretamente no website das empresas.

Como forma de complementar o levantamento realizado nos congressos também foram considerados casos compilados e apresentados na forma de relatórios nacionais, em levantamento pelo governo da Alemanha (PLATAFORM INDUSTRIE 4.0, 2017) e casos reportados pelo governo do Japão, compilados pela iniciativa Robot Revolution Initiative (RRI, 2017). Ao final do levantamento foram identificados 38 casos. Este procedimento de coleta resultou em casos de diversas áreas de atuação e com diferentes resultados, não comprometendo o procedimento de amostragem realizado. O procedimento de busca foi realizado entre 20 a 30 de abril de 2017. Tanto nos congressos quanto nos relatórios nacionais a coleta de dados e informações foi complementada utilizando fontes de dados secundárias, como o website da empresa e publicações relacionadas ao caso analisado.

\subsection{Análise dos dados}

Para cada caso de aplicação da Indústria 4.0 identificado foram realizados dois levantamentos: (i) as tecnologias da Indústria 4.0 que foram utilizadas no caso e (ii) o nível de maturidade da implementação da Indústria 4.0 identificado no caso analisado. As tecnologias de implementação apresentadas neste artigo foram definidas a partir da revisão de literatura apresentada na Tabela 1. A classificação do nível de maturidade foi realizada a partir da proposta desenvolvida por Porter e Heppelmann (2015). Apesar de as demais propostas apresentadas no referencial teórico deste artigo (SCHUMACHER et al., 2016; PWC, 2016; ROCKWELL AUTOMATION, 2014; LANZA et al., 2015) apresentarem uma avaliação mais abrangente e completa da implementação da Indústria 4.0, as informações relatadas nos casos analisados não possibilitam a identificação do nível de maturidade da aplicação reportada. Entre as propostas analisadas, a desenvolvida por Porter e Heppelmann (2014) está direcionada ao nível de maturidade da tecnologia implementada na solução (produto/serviço). Esta proposta foi utilizada em razão das fontes de evidência dos casos apresentarem indícios suficientes para a identificação do nível de maturidade da Indústria 4.0 identificados nesta proposta. Sendo assim, cada um dos casos de aplicação analisados é classificado de acordo com quatro níveis de maturidade tecnológica, definidas como: (i) monitoramento, (ii) 
controle, (iii) otimização e (iv) autonomia. Nesta proposta, a dimensão (i) monitoramento engloba empresas em que são aplicadas tecnologias capazes de monitorar e reportar informações do ambiente em tempo real, criando novos dados e informações. Empresas classificadas no nível (ii) controle possuem aplicações que podem controlar por meio de software locado internamente ou na nuvem o funcionamento de dispositivos. A dimensão (iii) otimização diz respeito à empresas que possuem algoritmos ou análises de dados capazes de otimizar a operação dos produtos, sua utilização e manutenção. Por fim, empresas classificadas no nível (iv) autonomia são aquelas em que seus produtos podem aprender, adaptar-se ao ambiente e as suas preferências, e operar sozinhos (PORTER; HEPPELMANN, 2015). Os procedimentos de classificação realizados foram baseados nas evidências apresentadas nos casos de aplicação da indústria 4.0 analisados. Os resultados obtidos são apresentados na seção seguinte.

\section{RESULTADOS}

Após análise e classificação dos casos, percebeu-se que para cada caso analisado a quantidade de tecnologias adotadas e as áreas da gestão de operação variavam, com empresas adotando até quatro tecnologias ao mesmo tempo, em até quatro áreas diferentes da gestão de operação. Por conta disso, algumas análises percentuais ficaram comprometidas.

\subsection{Tecnologias da Indústria 4.0}

Em todas as áreas de aplicação predominou o uso de tecnologias IoT, seguida com a utilização de sistemas Físico-Cibernéticos e da utilização de dispositivos móveis (Tabela 3). Um caso de uso de tecnologia IoT é o da Audi, empresa automobilística alemã. São utilizados sensores inteligentes para monitorar a precisão de seleção e montagem das peças. A tecnologia acompanha as etapas de montagem e oferece feedback aos trabalhadores. Outro exemplo é a empresa de TI, Fujitsu, que implementou em algumas plantas a prova de conceito (PoC) utilizando IoT. A empresa faz uso de computação na nuvem, cloud service e realiza gerenciamento de seus painéis e visualização de big data. Como resultado da utilização desses dados, reduzem o tempo gasto para engenharia e realizam uma detecção precoce de anormalidades, melhorando a qualidade de seus produtos e serviços.

Tabela 3 - Frequência de aplicação de tecnologias da Indústria 4.0

\begin{tabular}{|c|c|c|}
\hline Tecnologias da Indústria 4.0 & Frequência & Percentual \\
\hline IoT & 30 & $46,15 \%$ \\
\hline Sistemas Físico-Cibernéticos & 10 & $15,38 \%$ \\
\hline Dispositivos móveis & 8 & $12,31 \%$ \\
\hline Computação em nuvem & 6 & $9,23 \%$ \\
\hline Análise e processamento de dados & 4 & $6,15 \%$ \\
\hline Manufatura aditiva & 4 & $6,15 \%$ \\
\hline Realidade aumentada & 3 & $4,62 \%$ \\
\hline Total & 65 & $100 \%$ \\
\hline
\end{tabular}

O uso de sistemas Físico-Cibernéticos foi o segundo mais frequente nos casos analisados. Um caso a ser exemplificado é o da CHT, que aplica esses sistemas para controle de processo. $\mathrm{O}$ sistema registra todos os parâmetros do processo e o trabalhador no local de produção é capaz de monitorá-lo em tempo real com representações gráficas em interfaces homem máquina em rede. Outro caso é o da empresa de guindastes alemã Wolffkran, a qual equipou seus guindastes com o roteador industrial que pode ser controlado remotamente. Os dispositivos 
incorporados sinalizam automaticamente quando há uma falha ou mau funcionamento no equipamento, além de permitir manutenção preditiva, consequentemente a redução de custos. A aplicação desenvolvida pela Thyssenkrupp utiliza nos seus processos uma fusão entre o mundo físico e as redes de dados, criando um "sistema cibernético" durante a produção de peças para elevadores. São transportadas informações como número de série, de desenho, de peça e ID de cliente em um código de matriz de dados ao longo do ciclo de vida do produto. Com o uso dessa tecnologia, a empresa cita ganhos sustentáveis no gerenciamento de energia, novas soluções de manutenção, processos logísticos e serviços otimizados. Além de melhorias na qualidade, reduções de defeito e aumento de produtividade. Também foi identificada a utilização frequente de sensores inteligentes e tecnologias que reduzem, principalmente, a possibilidade de erros nos processos de montagem ou desenvolvimento de produtos.

\subsection{Nível de maturidade dos casos da Indústria 4.0 e sua contribuição para o gerenciamento de operações}

No âmbito de nível de maturidade da aplicação da indústria 4.0 nos casos analisados foi constatado o nível de maturidade monitoramento como predominante (Tabela 4). Esta concentração indica que as aplicações dos casos desta pesquisa estão direcionadas a monitorar e reportar informações do ambiente em tempo real, estado incipiente em relação ao nível de maturidade (PORTER; HEPPELMANN, 2015). O nível controle é mais identificado nos casos com aplicações nas áreas de gerenciamento da manufatura.

Tabela 4 - Nível de maturidade tecnológica dos casos avaliados em cada área da Gestão de Operações.

\begin{tabular}{|l|c|c|c|c|}
\cline { 2 - 5 } \multicolumn{1}{c|}{} & \multicolumn{4}{c|}{ Nível de maturidade de aplicações 4.0 } \\
\cline { 2 - 5 } \multicolumn{1}{c|}{} & Monitoramento & Controle & Otimização & Autonomia \\
\hline Casos de sucesso Indústria 4.0 & $29(76,3 \%)$ & $6(15,7 \%)$ & $2(5,2 \%)$ & $1(2,6 \%)$ \\
\hline
\end{tabular}

Já no nível de maturidade de otimização foram identificados dois casos. Um deles refere-se à empresa HBM, a qual oferece uma solução que permite o monitoramento e controle de toda a cadeia de medição na produção. Esse sistema tem aplicação nas áreas de Manufatura Just-inTime e Gestão da Qualidade; conta com algoritmos e cálculos críticos de qualidade que permitem identificar e corrigir problemas durante a produção. Para o nível de maturidade tecnológica autonomia foi identificado apenas um caso de aplicação, referente à empresa Stanley Black \& Decker. A empresa usa tecnologias IoT para manter o inventário de materiais e componentes o mais baixo possível, através de informações detalhadas e maior visibilidade de status e localização dos produtos. Além disso, os clientes também podem obter informações sobre o status de suas encomendas.

\section{CONSIDERAÇÕES FINAIS}

Este artigo tem por objetivo identificar as tecnologias e o nível de maturidade das aplicações da Indústria 4.0 nas empresas. Foram analisados 38 casos de sucesso, reportados em congressos da área e relatórios governamentais e de empresas, de implementação da Indústria 4.0 e suas contribuições nas diversas áreas do gerenciamento de operações das empresas. Foi verificado que as tecnologias IoT, sistemas Físico-Cibernéticos e dispositivos móveis são as mais utilizadas nas implementações da Indústria 4.0. A maior parte dos casos analisados ainda é classificada em um nível de maturidade incipiente, restrito a atividades de monitoramento.

Podem ser citadas duas principais contribuições da realização deste estudo. A primeira consiste na identificação da amplitude das contribuições da implementação da Indústria 4.0. Apesar de o nível de maturidade desta implementação nas empresas analisadas ainda estar em 
estágio inicial, são verificadas contribuições nas mais variadas áreas. Este resultado evidencia o caráter sistêmico da Indústria 4.0 nas empresas. Apesar de algumas conceituações da estarem restritas a troca de informações e autocontrole dos sistemas produtivos (WEYER et al., 2015), as aplicações que incorporam uma visão de integração da cadeia de valor (HERMANN et al., 2016) apresentam grande aderência a diversidade dos casos analisados. A segunda contribuição está direcionada a identificação das tecnologias empregadas na Indústria 4.0, permitindo estabelecer um panorama mais amplo sobre o impacto da implementação deste tipo de tecnologia nas empresas. Apesar de não se apresentar um roteiro de implementação da Indústria 4.0, o levantamento e apresentação de casos de sucesso permite um maior conhecimento sobre as potencialidades deste tipo de tecnologia e sua contribuição para as áreas de gerenciamento de operações. Por fim, as recomendações de trabalhos futuros estão direcionadas ao levantamento de mais casos de sucesso da implementação da Indústria 4.0, contribuindo para a disseminação das potencialidades de sua aplicação.

\section{REFERÊNCIAS}

ALMADA-LOBO, F. The Industry 4.0 revolution and the future of manufacturing execution systems (MES). Journal of Innovation Management, v.3, n.4, p.16-21, 2016.

BAGHERI, B.; YANG, S.; KAO, H. A.; LEE, J. Cyber-physical Systems Architecture for Self-Aware Machines in Industry 4.0 Environment. IFAC-PapersOnLine, v.48, n.3, p.1622$1627,2015$.

BRETTEL, M.; FRIEDERICHSEN, N.; KELLER, M.; ROSENBERG, M. How virtualization, decentralization and network building change the manufacturing landscape: An Industry 4.0 Perspective. International Journal of Mechanical, Aerospace, Industrial, Mechatronic and Manufacturing Engineering, v.8, n.1, p.37-44, 2014.

CNI - Confederação Nacional da Indústria. Indústria 4.0: novo desafio para a indústria brasileira, 2016. Disponível em:

http://www.portaldaindustria.com.br/relacoesdotrabalho/media/publicacao/chamadas/SondEs pecial_Industria4.0_Abril2016.pdf.

DRATH, R.; HORCH, A. Industrie 4.0: Hit or Hype?. IEEE Industrial Electronics Magazine, v.8, n.2, p.56-58, 2014.

HERMANN, M.; PENTEK, T.; OTTO, B. Design Principles for Industrie 4.0 Scenarios. Proceedings of 49th Hawaii International Conference on System Sciences (HICSS),

Koloa/HI, USA. pp. 3928-3937, 2016. Doi: 10.1109/HICSS.2016.488

HOZDIĆ, E. (2015). Smart Factory for Industry 4.0: A Review. International Journal of Modern Manufacturing Technologies, 2(1): 2067-3604.

KAGERMANN, H.; WAHLSTER, W.; HELBIG, J. Recommendations for Implementing the Strategic Initiative Industrie 4.0. Final report of the Industrie 4.0 Working Group, 2013.

LANZA, G.; HAEFNER, B.; KRAEMER, A. Optimization of selective assembly and adaptive manufacturing by means of cyber-physical system based matching. CIRP AnnalsManufacturing Technology, v.64, n.1, p.399-402, 2015.

LASI, H.; KEMPER, H.G. Industry 4.0. Business \& Information Systems Engineering, v.4, 2014. Doi: 10.1007/s12599-014-0334-4

LEE, I., LEE, K. The Internet of Things (IoT): Applications, investments, and challenges for enterprises. Business Horizons, v.58, n.4, p.431-440, 2015. 
LEE, J.; BAGHERI, B.; KAO, H. A. (2015). A cyber-physical systems architecture for industry 4.0-based manufacturing systems. Manufacturing Letters, v.3, p.18-23, 2015.

LIAO, Y.; DESCHAMPS. F.; LOURES, E. F. R.; RAMOS, L. F. P. Past, presente and future of Industry 4.0 - a systematic literature review and research agenda proposal. International Journal of Production Research, v.55, n.12, p.3609-3629, 2017.

LU, Y. (2017). Industry 4.0: A Survey on Technologies, Applications and Open Research Issues. Journal of Industrial Information Integration, In press, 2017.

PAULK, M.; CURTIS, B.; CHRISSIS, M.B.; WEBER, C.V. (1993). Capability Maturity Model for Software. Encyclopedia of Software Engineering, 1993.

PLATAFORM INDUSTRIE 4.0. Map of Industrie 4.0 use cases. Disponível em: <http://www.plattform-i40.de/I40/Navigation/EN/InPractice/Map/map.html>. Acessos nos dias: 24, 25, 26, 27, 28, 29, 30 de abr 2017.

PORTER, M. E., HEPPELMANN, J. E. How Smart, Connected Products are Transforming Competition. Harvard Business Review, 2015.

PWC - PricewaterhouseCoopers. Global Industry 4.0 Survey. Industry 4.0: Building the digital enterprise., 2016. Disponível em:https://www.pwc.com/gx/en/industries/industries4.0/landing-page/industry-4.0-building-your-digital-enterprise-april-2016.pdf

QIN, J., LIU, Y., GROSVENOR, R. A Categorical Framework of Manufacturing for Industry 4.0 and Beyond. Procedia CIRP, v.52, p.173-178, 2016.

ROCKWELL AUTOMATION. The Connected Enterprise Maturity Model. 2014. Disponível em: http://literature.rockwellautomation.com/idc/groups/literature/documents/wp/cie-wp002_en-p.pdf

RRI - Robotic Revolution Initiative. Map of Industrie 4.0 use cases. Disponível em: <http://usecase.jmfrri.jp/\#/en>. Acessos nos dias: 24, 25, 26, 27, 28, 29, 30 de abr 2017.

SCHUMACHER, A.; EROL, S.; SIHN, W. A Maturity Model for Assessing Industry 4.0 Readiness and Maturity of Manufacturing Enterprises. Procedia CIRP, v.52, p.161-166, 2016.

SHAFIQ, S. I.; SANIN, C.; SZCZERBICKI, E.; TORO, C. Virtual engineering object/virtual engineering process: a specialized form of cyber physical system for Industrie 4.0. Procedia Computer Science, v.60, p.1146-1155, 2015.

SHROUF, F.; ORDIERES, J.; MIRAGLIOTTA, G. Smart Factories in Industry 4.0: A Review of the Concept and of Energy Management Approached in Production Based on the Internet of Things Paradigm. Proceedings of do International Conference on Industrial Engineering and Engineering Management, 9-12 de dezembro 2014, Selangor Darul Ehsan, Malaysia.

STOCK, T.; SELIGER, G. Opportunities of sustainable manufacturing in Industry 4.0. Procedia CIRP, v.40, p.536-541, 2016.

TAMÁS, P.; B. ILLÉS; DOBOS, P. Waste reduction possibilities for manufacturing systems in the industry 4.0. Proceedings of IOP Conference Series: Materials Science and Engineering, v.161, n.1, 2016.

WEYER, S.; SCHMITT, M.; OHMER, M.; GORECKY, D. Towards Industry 4.0 Standardization as the Crucial Challenge for Highly Modular, Multi-vendor Production Systems. IFAC-PapersOnLine, v.48, n.3, p.579-584, 2015. 
ZUEHLKE, D. SmartFactory-Towards a factory-of-things. Annual Reviews in Control, v.34, n.1, p.129-138, 2010. 